\title{
A Rule-Based Functional-Structural Model of Rice Considering Source and Sink Functions
}

\author{
Lifeng Xu ${ }^{1,2,5}$, Michael Henke ${ }^{2,5}$, Jun Zhu ${ }^{1}$, Winfried Kurth ${ }^{2}$, Gerhard Buck-Sorlin ${ }^{3,4,5}$ \\ ${ }^{1}$ Institute of Bioinformatics, Zhejiang University, 310029 Hangzhou, P.R. China \\ ${ }^{2}$ Dept. Ecoinformatics, Biometrics and Forest Growth, Büsgen Institute, Georg-August- \\ University Göttingen, Büsgenweg 4, 37077 Göttingen, Germany \\ ${ }^{3}$ Wageningen UR, Centre for Crop Systems Analysis, Droevendaalsesteeg 1, 6708 PB \\ Wageningen, The Netherlands \\ ${ }^{4}$ Corresponding author: gerhard.buck-sorlin@wur.nl \\ ${ }^{5}$ These authors have contributed equally to this article
}

\begin{abstract}
As a first step towards a generic genotypephenotype model of rice, we present here a model of the growth and morphology of rice in combination with ecophysiological processes using the technique of functional-structural plant modelling (FSPM) and the interactive modelling platform GroIMP along with the graph-based Relational Growth Grammar formalism. The model constitutes a simple yet functionally coherent phenotype model of rice, consisting of a set of morphogenetic RGG rules describing an "average" developmental course and final morphology, partially linking yield traits to processes (tiller and grain number, stem length, grain filling rate, grain weight).
\end{abstract}

\section{Introduction}

Facing the challenge to meet increasing demands for food, rice (Oryza sativa L.) breeding research aims at both substantial and sustainable yield increase. This requires deeper understanding of the way ecophysiological characters functionally contribute to yield, and how genes interact with changing environments and act upon physiological processes contributing to yield. Yield formation in rice is a complex of (mainly developmental) processes concurrently taking place at different hierarchical scales and times and involving the action of genes, together with environmental and management factors, on physiological processes [1]. Despite the considerable investment in the descriptive characterisation of yield-related traits, rice research has still made insufficient progress with respect to a more profound comprehension of the physiological mechanisms involved in yield formation. Due to the complexity of such a system, a modelling approach is appropriate. Recent approaches taking meristem-based crop modelling concepts as their departure point, have been promising in terms of simulation of some of these processes [2].

Functional-structural plant models (FSPM), also referred to as virtual plants, are models that explicitly describe the development over time of the 3D architecture or structure of plants as governed by physiological processes which, in turn, are driven by environmental factors [3]. The FSPM approach applied to crops is thus the visual modelling and simulation in 3D of crop development using computer graphics in order to better capture, visualize, and quantify the phenology and morphogenesis of various crops under different conditions of growth. Some workers have attempted to link this with quantitative genes and metabolic regulatory networks $[4,5]$. Other workers successfully implemented ecophysiological and graphically realistic models of maize $[6,7]$, wheat $[8$, 9] and also rice [10, 11], mostly using L-system-based approaches $[12,13]$; in some of the mentioned instances, functional elements were added to model physiological processes (such as light interception or growth based on source availability and sink competition), e.g. the Greenlab model used by [7], in which a beta-function-based sink/source approach is used to model organ growth. Watanabe et al. [10] proposed a spatially explicit virtual rice model to specify plant architecture and to find appropriate functions to represent growth and development across all developmental stages. The authors found that this approach showed the potential to demonstrate the 
differences in structure and development between cultivars.

With the aim to provide improved tools for FSPM, especially the more intuitive integration of models for physiological functions and metabolic networks into structurally explicit models, the open-source interactive modelling platform GroIMP along with the extension and generalization of the string-based Lsystem formalism to the graph-based Relational Growth Grammars (RGG) formalism have been developed [4, 14]. This approach also allows the explicit representation of genotypes as objects, a feature which will be exploited in future versions of this model.

Here we will present an FSPM of rice, representing growth and morphology in combination with selected ecophysiological processes including photosynthesis and sink functions based on a common assimilate pool, and show first simulation results. The model thus produces a simple phenotype based on a set of morphogenetic rules describing an "average" developmental course and final morphology linking yield traits to selected physiological processes. This constitutes the first step of a model system of rice FSPMs, which will ultimately integrate information on QTLs, environments and their interaction with each other in regulatory networks.

\section{Materials and methods}

\subsection{Modelling language and platform}

The present rice FSPM was written using the eXtended L-System modelling language (XL) [14], an extension of L-systems [12, 13]. The modelling platform GroIMP (http://sourceforge.net/projects/ groimp/) was employed for model implementation and visualisation. The latter is designed as an integrated platform which incorporates modelling, visualisation and user interaction, and provides a plugin for XL. XL being a superset of Java ${ }^{\mathrm{TM}}$, it furthermore provides access to the entire functionality of Java ${ }^{\mathrm{TM}}$, thereby unifying the three programming paradigms imperative, rule-based and object-oriented in one modelling language [14].

\subsection{General features of the rice FSPM}

The rice FSPM is a rather extensive set of XL modules for 1) the general setup of the system (initiation of the plant individuals, possibly with a genotype object; initiation of output charts), 2) vegetative (leaf and internode development) and 3) generative morphology (panicle development and grain formation), 4) photosynthesis, 5) light interception (based on a Monte-Carlo radiation model described in [15]), 6) parameters (mainly environmental), 7) metabolic and 8) genetic regulation (the latter two currently optional), as described in [5] for barley. Only the most important rules will be described in the following (for a more extensive description of the general system see $[4,5])$.

In the following sections we will describe selected features of the current model, in which growth and development are based on source (local photosynthesis of assimilates and local storage in a central pool) and sink functions (reallocation of assimilates for growth as a function of sink strength, i.e. relative potential growth rate).

\subsection{Modelling of source activity}

In order to model source activity, we implemented the photosynthesis model LEAFC3 [16] in XL. It was parameterised for rice using data from the literature $[17,18,19]$. LEAFC3 is a generic model for the estimation of short-term steady-state fluxes of $\mathrm{CO}_{2}$, water vapour, and heat from leaves of $\mathrm{C} 3$ plant species, explicitly coupling all major processes involved in photosynthesis (biochemistry of assimilation process, stomatal conductance, leaf energy balance). This model has already been successfully used to model gas exchange in wheat by [20], and in barley (Ole Kniemeyer and Gerhard Buck-Sorlin, unpublished).

Input to the photosynthesis model was a weather file containing daily values of mean temperature, global radiation, and relative humidity. Since only daily totals of global radiation were available, the expected value for a given hour of the day was estimated using a sine function described in [21], assuming a daily average of atmospheric transmissivity, estimated as

$$
1-(s / d)
$$

where $s$ is the number of sunshine hours [h] and $d$ the daylength [h, from civil dawn to civil dusk].

To estimate local light interception and leaf photosynthesis, the radiation model of GroIMP was used. It is described in detail in [15]: The method is based on an inversed Monte-Carlo raytracer [22] and uses light sources and geometric objects placed into a scene. We used a single point light source with a power of $600 \mathrm{~W} \mathrm{~m}^{-2}$ (corresponding to incident global radiation, $1367 \mathrm{~W} \mathrm{~m}^{-2}$, attenuated by the atmosphere). To use the light model, an instance of it is invoked with two parameters: the total number of rays produced and traced from directly lit geometric objects to all the 
light sources in the scene, and the number of times a reflected or transmitted ray is traced (see Appendix A1).

A leaf blade is modelled as a collection of parallelogram objects of different size and orientation. Each leaf has a parameter $j$ which stores intercepted PAR (Photosynthetically Active Radiation). Once a leaf is formed, it is identified with a label and its absorbed PAR determined using a method getAbsorbedPower of the light model, which returns the spectrum of absorbed PAR. This spectrum is converted to Photosynthetic Photon Flux Density [ $\mu \mathrm{mol}$ PPFD m $\mathrm{m}^{-2}$ ] using a coefficient of 2.27 for daylight (Appendix A2).

The PSdaily() method which invokes the LEAFC3 model with input leaf area, temperature and PAR, is used to calculate daily assimilate production per leaf. The output of all leaves is at each daily step added up to a central assimilate pool (Appendix A3).

\subsection{Modelling of sink activity}

The timing and growth duration of active sinks drives the conversion of assimilates to harvestable dry matter, i.e. grain yield. In our FSPM approach the orchestration of sink activity is prescribed by growth and development rules, and the overall biomass production is an emergent property of the integration of these rules applied over time to simulated structures. In addition, the rate of extension of each organ is described as a beta growth function [23]. This function describes the dynamics of extension and biomass accumulation of organs: the application of this function to all organs over time then describes the growth of the whole plant.

The sink strength of a growing organ can be circumscribed by its potential growth rate, which is the derivative of the beta growth function proposed by Yin et al. [23]:

$$
c_{m}=w_{\max }\left(\frac{2 t_{e}-t_{m}}{t_{e}\left(t_{e}-t_{m}\right)}\right)^{\frac{t_{m}}{t_{e}-t_{m}}}
$$

where $c_{m}$ is the maximum growth rate in the linear phase, i.e. at time $t=t_{m}$, and $t_{e}$ is the time when growth ends (i.e. growth rate turns zero) as the maximum dimension or mass $w_{\max }$ is reached.

The potential growth rate $\left(s s_{p o t}\right)$ of an organ at time $t$ is then computed as:

$$
s S_{p o t}=\frac{d w}{d t}=c_{m}\left(\frac{t_{e}-t}{t_{e}-t_{m}}\right)\left(\frac{t}{t_{m}}\right)^{\frac{t_{m}}{t_{e}-t_{m}}}
$$

Global sink demand is defined as the sum of all potential growth rates of concurrently growing organs, multiplied with the modeled step size:

$$
s d_{t o t}=\sum s s_{p o t} \Delta t
$$

Multiplication of $s s_{r e l}$ with the current size of the common assimilate pool ap results in the realized growth rate $g r_{\text {real }}$, thereby making sure that this is not bigger than $s s_{\text {pot }}$ :

$$
g r_{\text {real }}=\frac{s s_{\text {pot }}}{s d_{\text {tot }}} \text { ap } \quad\left(g r_{\text {real }} \leq s s_{\text {pot }}\right)
$$

Once growth of an organ is realized with rate $g r_{\text {real }}$, the central assimilate pool is updated accordingly (Appendix A4).

Finally, a growth respiration term is considered in the form of a conversion factor ( $g$ glucose $\mathrm{g}^{-1} \mathrm{dm}$ ), which is proportional to growth rate as described in [21]. Likewise, maintenance respiration is computed as a fixed proportion $\left(0.014 \mathrm{~g}\right.$ glucose $\left.\mathrm{g}^{-1} \mathrm{dm}\right)$ of structural biomass [21]. Both terms are subtracted from the central pool at each step.

\subsection{Vegetative and generative development}

To simulate vegetative and generative development, a set of growth, developmental and branching rules is repetitively applied to a Meristem module and all its ensuing organs, leading to the visible phenotype. The structural framework created thus is then used to simulate and analyse the dynamics of assimilate flow as dictated by local (potential) growth rates and assimilate availability in the central pool.

Here, we describe the main rules for organ formation in words (some selected code can be found in Appendix A5): Formation of a new organ from a meristem occurs after some resting time (plastochron). This is done by counting an internal parameter pc down to zero. The main stem and tillers are created within the limits given by topological parameters (i.e. maximum rank and order). A new leaf is formed with an initial length and diameter. The meristem is reinitiated at the tip of the shoot, and the rank increased by one; at the same time the plastochron is set to its initial value (as specified by a global variable 
PLASTOCHRON). Other rules determine bending-up of the culm due to phototropism.

The potential extension and final dimension of organs (leaves, internodes, etc...) depend upon their rank and age, while the actually achieved dimensions are also a function of sink competition and assimilate availability, as described in section 2.4.

Leaf dimensions are determined using the beta growth function from [23], calculating dry matter increment as a function of time which is then converted into leaf area.

Once a temperature sum threshold $t_{g e n}$ is surpassed, the vegetative meristem is transformed into a generative meristem (Appendix A6), which is followed by grain primordia formation and grain filling (the latter again by using the beta growth function to describe the process). As long as the central assimilate pool is not exhausted, the generative meristem then recursively produces grains, thereby diminishing and usually quickly exhausting the assimilate pool.

\section{Results}

\subsection{Photosynthesis}

Fig. 1 shows the dynamics of net photosynthesis at hourly steps as simulated using LEAFC3 in XL, using a weather file for the IRRI wetlands weather station (Los Baños, Philippines) as an input. ${ }^{1}$ The LEAFC3 model produces a very regular diurnal pattern, for both net assimilation rate and stomatal conductance, for the period considered (May 19 $9^{\text {th }}$ - May 30, 2008 ). Simulated leaf temperature served as a control to see if the model was producing meaningful output.

Simulated net assimilation was between 20 and 25 $\mu \mathrm{mol} \mathrm{CO} \mathrm{m}^{-2} \mathrm{~s}^{-1}$ at a leaf temperature of about $25^{\circ} \mathrm{C}$. This fits well with the findings in [18].

\subsection{Dynamics of the central assimilate pool}

In the model, the central assimilate pool is updated as a function of local leaf photosynthesis and growth of an organ (Fig. 2). The dynamics of the central assimilate pool is characterised by three phases: after an initial decrease due to establishment growth and unfolding of seedling leaves, a strong increase during the mid to late vegetative phase can be observed. A sharp drop followed by a complete exhaustion of the

\footnotetext{
${ }^{1} \mathrm{http}$ ://beta.irri.org/index.php?option $=$ com content\&task=vie $\underline{\text { w\&id }=459 \& \text { Itemid }=328}$
}

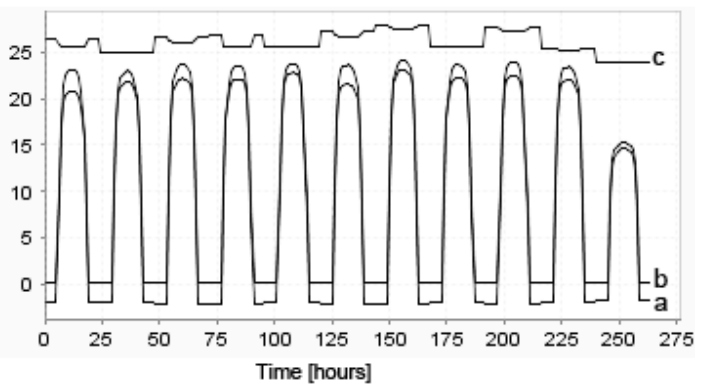

Fig. 1: Dynamics of net photosynthesis as simulated with a standalone version of LEAFC3, using a weather file for the IRRI wetlands weather station (Los Baños, Philippines) as an input. a) simulated net assimilation $\left[\mu \mathrm{mol} \mathrm{CO}_{2} \mathrm{~m}^{-2} \mathrm{~s}^{-1}\right]$, b) simulated stomatal conductance $\left[0.1 * \mathrm{mmol} \mathrm{CO} \mathrm{m}^{-2} \mathrm{~s}^{-1}\right]$, and c) simulated leaf temperature $\left[{ }^{\circ} \mathrm{C}\right]$. Step size: hourly. Time considered: from May 19 to May 30, 2008.

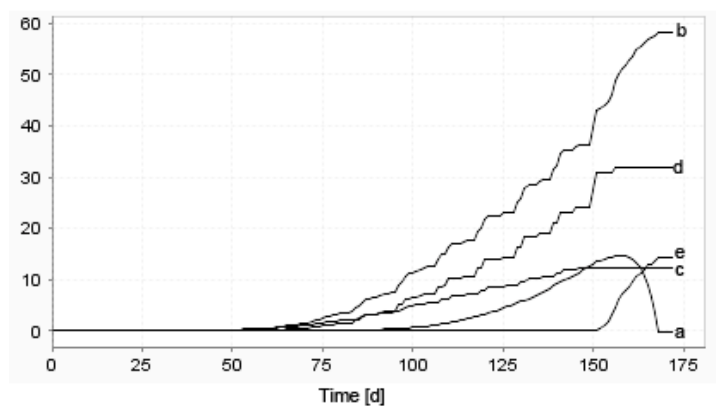

Fig. 2: Simulated dynamics of a) the central assimilate pool [g], b) total dry mass [g], as well as of the three organ types: c) leaves $[\mathrm{g}], \mathrm{d})$ internodes $[\mathrm{g}]$, and e) grains $[\mathrm{g}]$.

pool within a few steps indicates the onset and completion of the grain filling stage.

\subsection{Morphology of the rice FSPM}

This model reproduces plant architecture and morphology of rice from the seedling stage to maturity. Grain filling has not yet been fully implemented in this first step, but with the processes of leaf extension, internode elongation, and grain formation (constituting the sink model), the model already exhibits a reasonably faithful appearance at all growth stages. Two views on canopies of different phenological stages (late vegetative and post-flowering) are shown in Fig. 3. Morphological parameter sets are based on empirical data from the literature [11] and measurements (data not shown). With this implementation of a properly calibrated rice model, we obtain not only the 3D representation of the data sets but can also envisage to predict, e.g., variations of rice morphology under certain environmental conditions. 

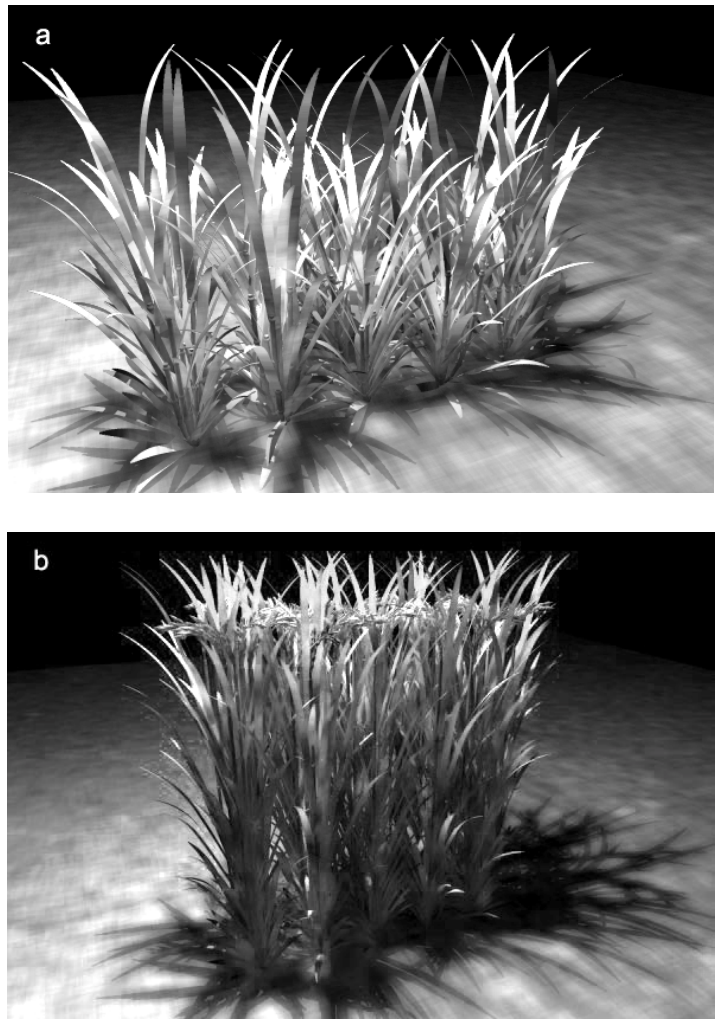

Fig. 3: Simulated rice morphology: small canopy, at two different developmental stages: a) late vegetative, and b) post-flowering. Scene rendered with the GroIMP Twilight renderer to show light distribution within the canopy, including backscattering of light rays from the ground.

\section{Discussion}

Existing data sets of rice have been processed and combined in this FSPM approach and it turned out that linking morphological and phenological appearance with ecophysiological functions plottable in dynamically updated graphs was very intuitive and informative.

As models become more mechanistic and comprehensive, crop-growth models can be used to mimic the genetic characteristics of plants [24]. Indeed, conventional crop growth models are now increasingly used in breeding programmes to assist in the design of new plant types [24, 25]. Modelling not only plays a role in data synthesis and quantitative prediction, but certainly also in heuristics and system design, future roles of modelling proposed by [1] for 'crop systems biology'. Modelling approaches like the present one can be an intuitive and extensible tool to enhance our understanding of complex crop phenotypes, which will ultimately lead to new and improved crop cultivars.

Although the present model is still at an early stage with respect to the complexity of physiological processes considered, it already contains the main elements necessary to extend it to a largely generic genotype-phenotype model, which is the ultimate goal of our study.

The photosynthesis model and the source-sink model are the two main models of the present rice FSPM. In the case of crop plants, growth and development are often not limited by source availability (source being abundant), but driven, respectively, limited, by sink activities [26]. However, most crop models are based on the simulation of photosynthesis and make very simplified assumptions about sink activity of the different organ types. If those models are applied to interpret data containing quantitative genetic variability in yield formation, the outcome could possibly just reflect that part of variability that directly links to the photosynthesis process, whilst masking or neglecting other, sinkrelated aspects of yield formation.

The beta growth function [23] is particularly suitable to describe the dynamics of sinks (Xinyou Yin, pers. comm.). Using it extensively in our sink model, we intend to test it further for its sensitivity to environmental and genetic factors.

An interesting virtual plant study on the architecture of different hybrid rice cultivars has been conducted by Zheng et al. [11]: Digitizing four developmental stages from panicle initiation to the grain-filling stage, extracting the structural information obtained thus, then computing the light interception and potential carbon gain of these virtual plants, they could show that certain plant types with steeper leaf divergence angles exhibited a higher light penetration of the canopy when sun elevation was high. The result obtained was, however, also related to leaf area index. This study already shows the enormous potential use of a simple virtual plant (i.e. just constituting digitized 3D structural information).

As outlined in the introduction, the present work is but the initial step of a modelling project, ultimately aiming at linking physiological processes and quantitative genetics of aspects of yield formation (QTLs for grain and tiller number), and the feedback among them, with a realistic 3D structural phenotype model of rice. In one of the next steps, the sink model will be refined to properly simulate grain filling: More specifically, this will involve splitting the process up into two successive processes, determination of potential grain number, followed by grain filling proper. Promising approaches for this can be found in [18]. More recently, [27] captured nitrogen distribution in the wheat plant during grain-filling using a processed-based approach. It remains to be carefully evaluated if the present rice FSPM should be enlarged to also include a module for nitrogen uptake and 
transport, as has been partly achieved for barley (Katarína Smoleňová, personal communication) or for rapeseed [28]. Having in mind the ultimate objective of making better sense of quantitative genetic variation in grain yield, it is difficult to decide a priori which physiological processes to include and which to neglect. A truly mechanistic modelling approach would certainly strive at maximizing the number of modelled processes. However, calibration of such an extensive model often turns into a very timeconsuming task [29].

To fulfill the future roles of crop modelling in its wider context of crop systems biology [1], it is important to understand the physiological and morphological reaction norm (with respect to growth and development) of the rice plant through its entire life cycle at different scale levels (organ, plant, canopy). The present rice FSPM could be considered as the first step towards obtaining a tool for knowledge integration and visualisation in the sense of crop systems biology.

\section{Acknowledgments}

L.X. received a six-month young researcher scholarship (A/08/96297) from the German Academic Exchange Service (DAAD, http://www.daad.de), enabling him to conduct predoctoral studies on modelling in the work group of W.K.. This research was partially supported by the National Basic Research Program of China [2004CB117306] and the National High Technology Research and Development Program of China [2006AA10A102]. The financial support is gratefully acknowledged.

\section{References}

[1] X. Yin and P. Struik, "Applying modelling experiences from the past to shape crop systems biology: the need to converge crop physiology and functional genomics", The New Phytologist, 2008, 179(3), pp. 629-642.

[2] D. Luquet, A. Clément-Vidal, D. Fabre, D. This, N. Sonderegger, and M. Dingkuhn, "Orchestration of transpiration, growth and carbohydrate dynamics in rice during a dry-down cycle", Functional Plant Biology, 2008, 35 , pp. 689-704.

[3] J. Vos, L.F.M. Marcelis, P.H.B. de Visser, P.C. Struik, and J.B. Evers, Functional-structural plant modelling in crop production, Springer, New York, 2007.

[4] G.H. Buck-Sorlin, O. Kniemeyer, and W. Kurth, "Barley morphology, genetics and hormonal regulation of internode elongation modelled by a relational growth grammar", New Phytologist, 2005, 166, pp. 859-867.
[5] G.H. Buck-Sorlin, R. Hemmerling, O. Kniemeyer, B. Burema, and W. Kurth, "A rule-based model of barley morphogenesis, with special respect to shading and gibberellic acid signal transduction", Annals of Botany, 2008, 101, pp. 1109-1123.

[6] C. Fournier and B. Andrieu, "A 3D architectural and process-based model of maize development", Annals of Botany, 1998, 81, pp. 233-250.

[7] Y. Ma, M. Wen, Y. Guo, B. Li, P.-H. Cournede, and P. de Reffye, "Parameter optimization and field validation of the functional-structural model GREENLAB for maize at different population densities", Annals of Botany, 2008, 101, pp. 1185-1194.

[8] J.B. Evers, J. Vos, C. Fournier, B. Andrieu, M. Chelle, and P.C. Struik, "Towards a generic architectural model of tillering in Gramineae, as exemplified by spring wheat (Triticum aestivum)", The New Phytologist, 2005, 166, pp. 801-812.

[9] J.B. Evers, J. Vos, B. Andrieu, M. Chelle, and G.H. BuckSorlin, "Functional-structural plant modelling, applied to tillering in wheat", Italian Journal of Agronomy, 2008, 3, pp. 739-740.

[10] T. Watanabe, J.S. Hanan, P.M. Room, T. Hasegawa, H. Nakagawa, and W. Takahashi, "Rice morphogenesis and plant architecture: measurement, specification and the reconstruction of structural development by $3 \mathrm{D}$ architectural modelling", Annals of Botany, 2005, 95, pp. 1131-1143.

[11] B. Zheng, L. Shi, Y. Ma, Q. Deng, B. Li, and Y. Guo, "Comparison of architecture among different cultivars of hybrid rice using a spatial light model based on 3-D digitizing”, Functional Plant Biology, 2008, 35, pp. 900-910.

[12] P. Prusinkiewicz and A. Lindenmayer, The Algorithmic Beauty of Plants, Springer-Verlag, New York, 1990.

[13] W. Kurth, "Morphological models of plant growth. Possibilities and ecological relevance", Ecological Modelling, 1994, 75/76, pp. 299-308.

[14] O. Kniemeyer, Design and Implementation of a Graph Grammar Based Language for Functional-Structural Plant Modelling, Ph.D. thesis, BTU Cottbus, 2008.

[15] R. Hemmerling, O. Kniemeyer, D. Lanwert, W. Kurth, and G.H. Buck-Sorlin, "The rule-based language XL and the modelling environment GroIMP illustrated with simulated tree competition", Functional Plant Biology, 2008, 35(9-10), pp. 739-750.

[16] N.T. Nikolov, W.J. Massman, and A.W. Schoettle, "Coupling biochemical and biophysical processes at the leaf level: an equilibrium photosynthesis model for leaves of C3 plants", Ecological Modelling, 1995, 80, pp. 205-235.

[17] X. Yin, M. Van Oijen, and A.H.C.M. Schapendonk, "Extension of a biochemical model for the generalized stoichiometry of electron transport limited C-3 photosynthesis", Plant Cell and Environment, 2004, 27(10), pp. 1211-1222. 
[18] X. Yin and H.H. van Laar, Crop Systems Dynamics: An ecophysiological simulation model for genotype-byenvironment interactions, Wageningen Academic Publishers, Wageningen, 2005.

[19] A. Borjigidai, K. Hikosaka, T. Hirose, T. Hasegawa, M. Okada, and K. Kobayashi, "Seasonal changes in temperature dependence of photosynthetic rate in rice under a free-air $\mathrm{CO}_{2}$ enrichment", Annals of Botany, 2006, 97, pp. 549-57.

[20] J. Müller, P. Wernecke, and W. Diepenbrock, "LEAFC3$\mathrm{N}$ : A nitrogen-sensitive extension of the $\mathrm{CO}_{2}$ and $\mathrm{H}_{2} \mathrm{O}$ gas exchange model LEAFC3 parameterised and tested for winter wheat (Triticum aestivum L.)", Ecological Modelling, 2005, 183, pp. 183-210.

[21] J. Goudriaan and H.H. van Laar, Modelling Potential Crop Growth Processes, Kluwer Academic Publishers, Dordrecht, The Netherlands, 1994.

[22] E. Veach, Robust Monte Carlo Methods for Light Transport Simulation, Ph.D. thesis, Stanford University, 1998

[23] X. Yin, J. Goudriaan, E.A. Lantinga, J. Vos, and H.J. Spiertz, "A Flexible Sigmoid Function of Determinate Growth", Annals of Botany, 2003, 91, pp. 361-371.

[24] X. Yin, M.J. Kropff, and P. Stam, "The role of ecophysiological models in QTL analysis: the example of specific leaf area in barley”, Heredity, 1999, 82, pp. 415-421.

[25] G.L. Hammer, M. Cooper, F. Tardieu, S. Welch, B. Walsh, A. van Eeuwijk, S.C. Chapman, and D. Podlich, "Models for navigating biological complexity in breeding improved crop plants", Trends in Plant Science, 2006, 11, pp. 587-593.

[26] T. Fourcaud, X. Zhang, A. Stokes, H. Lambers, and C. Körner, "Plant growth modelling and applications: the increasing importance of plant architecture in growth models", Annals of Botany, 2008, 101, pp. 1053-1063.

[27] J. Bertheloot, B. Andrieu, C. Fournier, and P. Martre, "A process-based model to simulate nitrogen distribution within wheat (Triticum aestivum) during grain filling", Functional Plant Biology, 2008, 35, pp. 781-796.

[28] C. Groer, O. Kniemeyer, R. Hemmerling, W. Kurth, H. Becker, and G.H. Buck-Sorlin, "A dynamic 3D model of rape (Brassica napus L.) computing yield components under variable nitrogen fertilisation regimes", Proceedings of FSPM 2007, Napier, NZ, 2007. http://algorithmicbotany.org/FSPM07/Individual/4.pdf

[29] G.H. Buck-Sorlin, O. Kniemeyer, and W. Kurth, "A functional-structural model of barley including genetic control and metabolic networks", In: J. Vos, L.F.M. Marcelis, P.H.B. de Visser, P.C. Struik, J.B. Evers (eds), Functionalstructural plant modelling in crop production, New York, 2007, chapter 21, pp. 243-252.

\section{Appendix}

\section{A1. Invocation of light model}

LightModel rad $=$ new LightModel $(1000000,10)$;

The total number of rays is 1000000 per step, a reflected or transmitted ray is traced 10 times.

\section{A2. Local PAR interception by each leaf (1f)}

lf $[j]=$

rad.getAbsorbedPower (lf) . integrate ( ) *GRPAR;

The method getAbsorbedPower of rad (an instance of LightModel created earlier, see A1) returns the spectrum of absorbed PAR. The coefficient GRPAR (2.27 for daylight) is used to convert this spectrum to Photosynthetic Photon Flux Density.

A3. Local assimilate pool (locPool) of a leaf (1f) and central assimilate pool (Cpool) of plant

lf.locPool = PSdaily (lf.c, time, j);

The three input parameters for PSdaily() are: leaf area, time, and PAR intercepted by this leaf, $j$.

For the central assimilate pool, the locPool of all leaves flagged as actively growing (isGrowing ()) are queried, the values are summed up and added into the central assimilate pool:

Cpool $+=$

sum( (* a:Leaf, (a.isGrowing ()) *).locPool ()) ;

A4. Updating of the central assimilate pool according to the realized growth of organs

Cpool -= glu_growth;

where glu_growth is the glucose converted from the biomass consumed by the growth of organs during one step.

\section{A5. Rules for organ formation (simplified)}

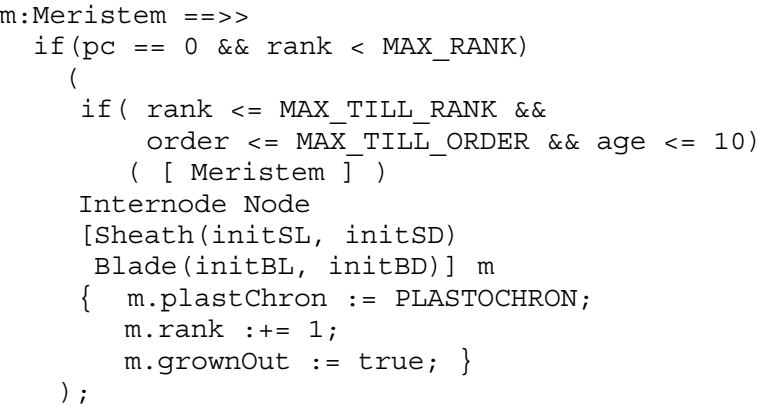

When the internal parameter pc (plastochron) is zero, a new phytomer is formed, consisting of an 
internode, a node, a leaf (with sheath and blade), as well as a lateral meristem. Formation of this lateral meristem, and subsequent outgrowth into a tiller, is restricted to a certain maximum tillering rank and order, MAX_TILL_RANK and MAX_TILL_ORDER. initSL/initBL and initSD/initBD are the initial values for the length and diameter of the new leaf (Sheath and Blade, respectively). The same meristem $m$ that created the phytomer, is reinserted at the tip of the shoot and equipped with a new value for the plastochron (the global variable plastchron, in degree-days) and a rank incremented by one.

\section{A6. Grain formation}

Transformation of the vegetative Meristem into a generative InflMeristem:

Meristem ==> if (tsum $>=$ tgen)

$$
\text { (Internode InflMeristem); }
$$

where tgen is the temperature sum threshold.

Formation of the grain:

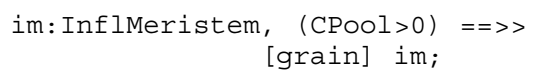

where a new grain (grain) is formed as long as the central assimilate pool (CPOOl) is not exhausted to zero and the grain forming meristem (im) is reinserted again at the tip. 